\title{
Gest Editorial
}

[to be published in Journal of New Music Research 2003, Vol. 32, No. 1, pp. 1-2]

The term "content processing" has already been around for some time but only in the last few years it has gained a clear meaning within the music research community. The articles presented in this special issue on Audio Content Processing refer to concepts such as content analysis, content browsing, content indexing, or content transformation. With these terms they address the problem of automatically generating and processing musical information from audio signals. They all share the idea that music processing requires a level of abstraction higher than the audio signal and the goal of automatically generating the content information, the metadata, to be processed.

Beyond music and audio, the enormous amount of unstructured multimedia data available nowadays and the spread of its use as a data source in many applications are introducing new challenges to researchers in information and signal processing. It is clear that the value of such information depends on how easily it can be found, retrieved, accessed, filtered and managed. The continuously growing amount of this digital multimedia information (available for example in digital archives, in the World Wide Web, in broadcast data-streams and in personal and professional databases, to name a few) increases the difficulty of its access and management, thus hampering its practical usefulness. Thus, the current challenge is the automatic processing of information based on content, permitting an easy and intuitive access to all this multimedia information.

Within the music research community Music Information Retrieval, which could be considered a term broader than Audio Content Processing, has quickly become a major focus of attention. This is clearly shown by the exponential growth of the International Conference on Music Information Retrieval (ISMIR) and the number of articles published in this topic just in the last few years. However, given this fast growth there are not many up to date articles looking into the field with some perspective. With the risk of becoming outdated quickly, in this special issue, we have put together a group of articles covering some basic topics within MIR. In particular, our aim has been to present extended overview articles in key areas of audio content processing.

In "Automatic Classification of Musical Instrument Sounds" by P. Herrera-Boyer, G. Peeters, and S. Dubnov, an exhaustive review of research on automatic classification of sounds from musical instruments is presented. The article focuses in one of the basic audio features used for sound description, musical timbre.

In "Melody Description and Extraction in the Context of Music Content Processing" by E. Gómez, A. Klapuri, and B. Meudic, the authors review the different techniques that have been proposed for melodic description and extraction. They focus on the issue of fundamental frequency detection and its musical structuring into melodies. 
In "Towards Rhythmic Content Processing of Musical Signals: Fostering Complementary Approaches" by F. Gouyon, and B. Meudic, the issue of rhythm is discussed in the context of music processing applications. Together with melody, the rhythm, is one of the most basic musical descriptors, and a very valuable one for many applications.

In "Audio Watermarking and Fingerprinting: For Which Applications?" by Gomes, P. Cano, E. Gómez, M. Bonnet, and E. Batlle, there is a discussion of two very successful technologies in the Audio Content Processing field. Watermarking and Fingerprinting techniques, originally developed for copyright protection applications, are shown to be not only mature techniques for audio identification but they also have a huge potential for new applications.

In "Representing Musical Genre: A State of the Art" by J. J. Aucouturier and F. Pachet, the authors discuss the various approaches in representing musical genre, a crucial metadata for the description of music content. It is shown that genre is intrinsically ill-defined and that we are still far from being able to automatically identify the genre of a piece of music.

Finally, in "Content-based Transformations" by X. Amatriain, J. Bonada, A. Loscos, J. LL. Arcos, and V. Verfaille, the authors go beyond the goal of content description, proposing new ideas in the area of the so-called content-based transformations. The concentrate in how to use the content information for transforming a sound in a more musically meaningful way.

Given that the research described in several of the articles of this special issue was partially funded by the IST European project CUIDADO, it is appropriate to briefly present it. The partners of the project are: IRCAM (coordinator), Sony CSL, Oracle, CreamWare, Universitat Pompeu Fabra, BenGurion University and Artpages International AS. The project aims to develop technologies for content-based products and services using the MPEG 7 emerging standard with an innovative and comprehensive content-based approach. The project covers content information extraction as well as exploitation: from the analysis process (extraction of descriptors), through the navigation process (retrieval methods and interfaces), up to the creative process (consuming and authoring tools). The scientific goal is to show how content-based technologies are not only for retrieval but also for representation and processing. The CUIDADO project is one particular example of the research interest and application potential of the audio content processing technologies. Many more research project examples can be identified.

In this especial issue we have tried to present a broad perspective of some key music research issues within an emerging field. I am confident that the reader will be able to grasp the great interest that all these topics have, the great number of wide open questions that still need to be answered, and the huge potential of the technologies that are being developed. 